\title{
Method of Evaluation of Forestry Machine Roll-over Protective Structure Efficiency
}

\author{
Alexander V. Pitukhin ${ }^{1, a^{*}}$, Igor G. Skobtsov ${ }^{1, b}$, Veniamin N. Shilovskiy ${ }^{1, c}$ \\ Oksana L. Dobrynina ${ }^{1, d}$ \\ ${ }^{1}$ Petrozavodsk State University, Lenin str., 33, Petrozavodsk, 185910, Russian Federation \\ apitukhin@petrsu.ru, biskobtsov@mail.ru, cshisvetnik@narod.ru, doksdobr@mail.ru
}

Keywords: Roll-over protective structure, Finite element method, Variable elasticity parameters

\begin{abstract}
Current standards in the field of self-propelled machinery for forestry require equipping tractor cabins with roll-over protective structures (ROPS). It is necessary to reduce the risk of operator's injuries caused by tractor rollovers or falling trees. This study deals with the investigation of forest machine ROPS deflected mode during lateral loading by using mathematical modeling. Finite element method and method of variable parameters of elasticity are taken as a principle of this work. One of regulatory materials requirements is the ability to absorb certain amount of potential energy during ROPS deformation. The estimation of the quantity of absorbed energy was made by the idealized stress-strain curve. The safety compliance assessment included the comparison between the quantity of this energy and its standard value. It can improve protection properties at the design stage. Ways of increasing ROPS efficiency are presented at the last part of the paper.
\end{abstract}

\section{Introduction}

Forest machine operator's activity is connected with the risk of injuries caused by tractor rollovers or falling trees. This is one of the major sources of fatal injury in the forest industry. Current standard ISO 8082-1 regulates static laboratory research of roll-over protective structure efficiency according to the safety requirements and test methods for machines and tractors for forestry. In some cases this research can be time-consuming, rather expensive and often technically difficult to perform because it requires testing full-scale samples of ROPS in a special laboratory.

Another way of ROPS efficiency evaluation is theoretical determination of protective frame deflected mode by using a mathematical model, and few researchers have addressed to this method. For example, Harris et al [1, 2], Powers et al [3], Etherton et al [4] analyzed agricultural tractor rollovers using finite element modeling. Dumitrache P. [5] investigated the behavior of earthmoving machine protective structures under load by using the method of parametric modeling. However, although these methods allow choosing the optimal parameters of ROPS at the stage of design, little attention has been paid to application of the plasticity modeling for estimation of strain energy value under plastic deformation.

\section{Estimation of ROPS Deflected Mode}

The methodology of estimation of forest machine roll-over protective structure deflected mode includes several steps. The estimation is carried out under condition of static loading. The initial direction of loading shall be horizontal and perpendicular to a vertical plane through the machine's longitudinal centerline. The whole load acts upon ROPS, i.e. we neglect the influence of tractor frame, windscreen glasses, coupling bars and other elements. The protective frame is presented in the form of core design using rectangular pipes as structural elements. This allows one to analyze ROPS behavior under the action of functional load (lateral force) using the finite element method.

1. Creation a finite-element model with the applied lateral force F (Fig. 1). 
2. Solution of the elastic problem in the first approximation, i.e. the estimation of ROPS deflected mode is performed by the finite element method excluding plastic deformations. Therefore we calculate the stress intensity of the first approximation $-\sigma_{i}^{I}$.

3. Determination of variable elasticity parameters according to the method, widely used in theory of plasticity [6].

$$
\begin{aligned}
& E^{*}=E \frac{3 \varphi}{2(1+\mu)+(1-2 \mu) \varphi} ; \\
& \mu^{*}=\frac{(1+\mu)-(1-2 \mu) \varphi}{2(1+\mu)+(1-2 \mu) \varphi},
\end{aligned}
$$

where, $E=$ Young's modulus of steel (steel modulus of elasticity);

$\mu=$ Poisson's ratio of steel;

$\varphi=$ plasticity function, depending on plastic deformation features,

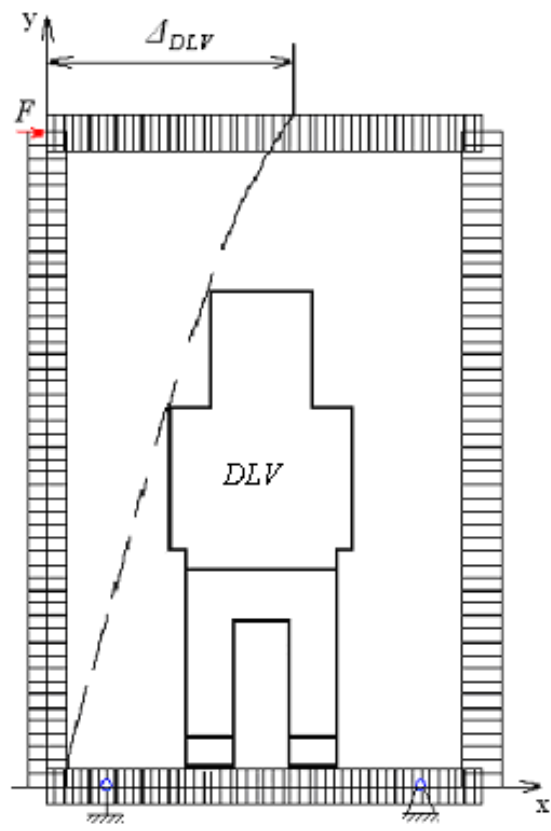

Fig. 1 Finite-element model of ROPS with DLV (deflection-limiting volume) combination.

$$
\varphi=\frac{\bar{\sigma}_{i}}{\bar{\varepsilon}_{i}}
$$

where, $\bar{\sigma}_{i}=\frac{\sigma_{i}}{\sigma_{y}}-$ relative stress intensity;

$$
\begin{aligned}
& \bar{\varepsilon}_{i}=\frac{\varepsilon_{i}}{\varepsilon_{y}}-\text { relative strain intensity; } \\
& \sigma_{i}, \varepsilon_{i}-\text { intensities of stress and strain. }
\end{aligned}
$$

We carried out calculations with the help of carbon steel idealized stress-strain curve (diagram) in coordinates $\sigma-\varepsilon$ (Fig. 2). According to this, the equations of plasticity function are

$$
\begin{array}{lll}
\varphi=\operatorname{tg} \gamma & \text { if } & \sigma_{i}>\sigma_{y} ; \\
\varphi=\operatorname{tg} \alpha & \text { if } & \sigma_{i} \leq \sigma_{y} ;
\end{array}
$$




$$
\operatorname{tg} \beta=\frac{E_{1}}{E},
$$

where, $E_{l}, E=$ steel modulus of elasticity, corresponding to the diagram plot (Fig. 2).

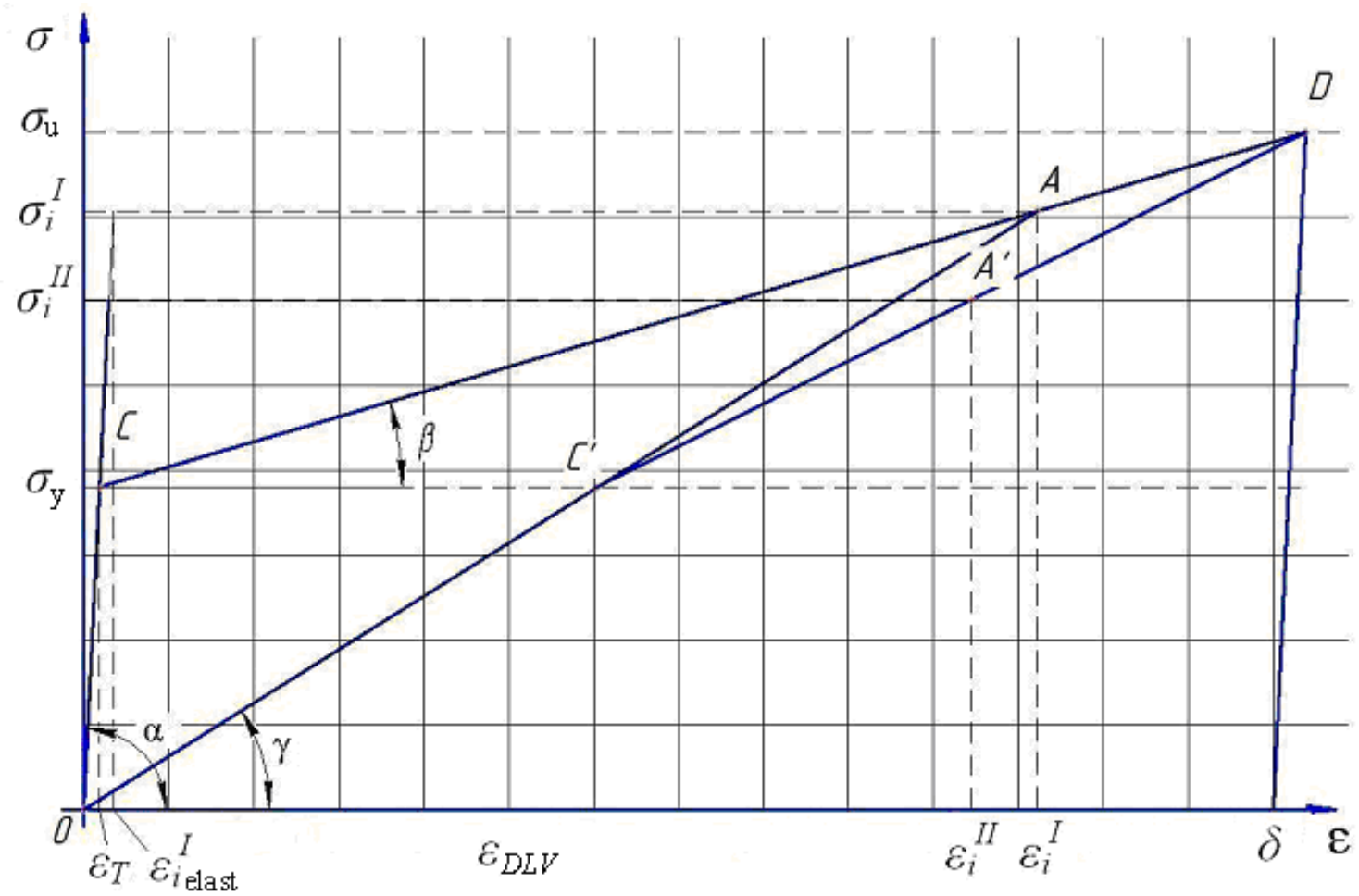

Fig. 2 Idealized stress-strain diagram.

Therefore we obtain

$$
\varphi=\left\{\begin{array}{c}
1, \sigma_{i} \leq \sigma_{y} \\
\frac{\bar{\sigma}_{i} \cdot \operatorname{tg} \beta}{\bar{\sigma}_{i}-1+\operatorname{tg} \beta}, \sigma_{i}>\sigma_{y} .
\end{array}\right.
$$

4. According to the calculated values of $E^{*}$ and $\mu^{*}$, we again solve the elastic problem, calculate the stress intensity of the second approximation $\sigma_{i}^{I I}$ and obtain new values of variable elasticity parameters.

The amount of approximations depends on the required accuracy of calculations.

5. Calculation of limiting deformation $\triangle \mathrm{DLV}$ (the point of contact between ROPS and DLV) is made by combining the model of DLV with the finite-element model of ROPS; DLV dimensions are in accord with ISO 3164.

6. Estimation of the value of strain energy UDLV, absorbed by ROPS during lateral loading at the moment when the construction under the load reaches the point of DLV is made by the stressstrain curve $\sigma-\varepsilon$. Therefore we can determine the value of strain energy UDLV, according to the value of $\varepsilon D L V$. The area under the stress-strain diagram $\sigma-\varepsilon$ equals the absorbed lateral energy.

7. The last step of our research is to compare the value of UDLV with the standard requirement of absorbed energy U, calculated according to ISO 8082. If

$$
U_{D L V} \geq U,
$$

the ROPS meets the standard safety regulations. 


\section{Experiment}

Materials. 1) We estimate the efficiency of TLK-4-01 wheeled skidder ROPS (Onega Tractor Plant, Russia). Machine TLK-4-01 mass is $M=14500 \mathrm{~kg}$, it's equipped with ROPS made from carbon steel that has the following mechanical characteristics: tensile strength $\sigma_{\mathrm{u}}=800 \mathrm{MPa}$; lower yield point $\sigma_{y}=380 \mathrm{MPa}$; direct strain $\delta=14 \%$. 2) Stress machine (press) IP - 1000-type (limit load $1000 \mathrm{kN}$ ) was used for experimental testing. The experimental values of absorbed strain energy are presented in Table 1.

Methods. Four groups of ROPS samples were made by different welding operators to avoid systematic error. After analyzation and calculation, we can obtain statistical values: sample mean, dispersion, standard deviation and so on. The results are shown in Table 1.

Table 1. Quantity of absorbed strain energy [J]

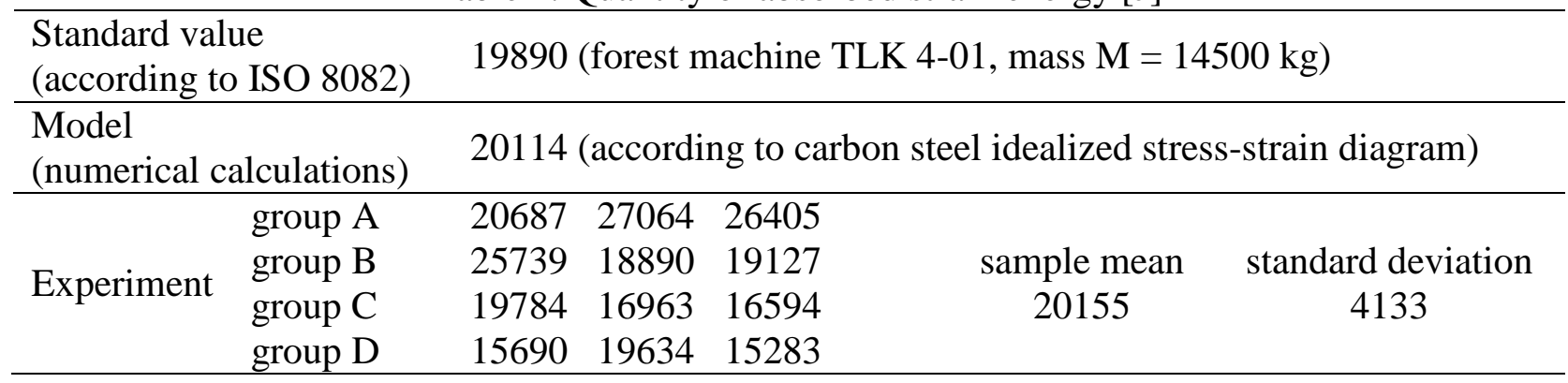

As shown, in some cases (for example, in the case of presence of crack-like defects in the maximum stress action zone $[7,8]$ ) the condition (Eq. 5) was not be fulfilled.

\section{Ways of Increasing ROPS Efficiency}

There are three ways to improve tractor cabin protection properties. The first direction is the solution of an optimization problem to choose substantiated structural parameters (geometrical dimensions, tolerances, defect sizes etc). The statement of an optimal design problem with the catastrophe theory application was presented in [9, 10]. The second way to increase ROPS efficiency is to investigate the availability of new perspective materials to produce protective constructions. And, the last way is connected with the application of additional safety devices. The article [11] contains the design of energy-absorbing cab support to provide additional energy absorption effect in case of rollover. Therefore, the protective effect is attained by plastic strain of cab support elements to ensure the safety of operator during an accidental roll-over.

\section{Conclusions}

Forest machine cabins have technical distinguishing features and differ from agricultural or earth-moving machine cabins due to their specific work conditions. The normative document in the field of self-propelled machinery for forestry establishes reproducible means of evaluating the force - deformation characteristics of ROPS obtained by experimental research in a special laboratory. Existing theoretical methods use different modeling approaches in order to study ROPS behavior. Although these methods apply to finite element method for estimation ROPS efficiency, they pay little attention to the determination of strain energy value under plastic deformation by lateral force.

In this paper we investigated the behavior of carbon steel ROPS under lateral load by using finite element modeling. The approach we have used in this study bases on the calculation of the variable parameters of elasticity with the help of carbon steel idealized stress-strain diagram. According to the methodical way of estimation of wheeled skidder ROPS deflected mode, we determined the value of strain energy $U_{D L V}$ at the moment when the construction under the load reaches the point of deflection-limiting volume. The experimental data suggests that, although the comparison between the quantity of this energy and its standard value showed that the size of this criterion meets the 
standard requirements, in some cases this condition will not be fulfilled.

\section{Acknowledgements}

This work was supported by the Strategic Development Program of Petrozavodsk State University (2012-2016).

\section{References}

[1] J. R. Harris, M. Ronaghi, K.A. Snyder, Analyzing tractor rollovers using finite element modeling, Anal. Solutions. 2(4) (1998) 24-25.

[2] J. R. Harris, V. H. Mucino, J. R. Etherton, Finite element modeling of rollover protective structures in static testing and rear overturns, J. Agric. Safe. Health. 6 (2000) 215-225.

[3] J. R. Powers, J. R. Harris, J. R. Etherton, M. Ronaghi, K. A. Snyder, T. J. Lutz, B. H. Newbraugh, Preventing tractor rollover fatalities: performance of the NIOSH AutoROPS, Injury Prevention. 7 (2001) 54-58.

[4] J. R. Etherton, R. G. Cutlip, J. R. Hams, M. Ronaghi, K. H. Means, A. Gillkpie, Static load test performance of a telescoping structure for an automatically diployable ROPS, J. Agric. Safe. Health. 8(1) (2002) 119-126.

[5] P. Dumitrache, Parametric modeling of the (ROPS/FOPS) protective structures geometry in order to study of their behavior using finite element method, The Sorging Journal. 6, 2 (2011) 9-12.

[6] I. A. Birger, R. R. Mavlutov, Strength of materials, Nauka, Moskow, 1986.

[7] A. V. Pitukhin, Optimal Design Problems Using Fracture Mechanics Methods, Comput. Struct. 65(4) (1997) 621-624.

[8] A. V. Pitukhin, Fracture Mechanics and Optimal Design, Int. J. Numer. Meth. Eng. 34(3) (1992) 933-940.

[9] A. V. Pitukhin, I. G. Skobtsov, The Statement of Optimal Design Problem with the Cusp Catastrophe Theory Application, Appl. Mech. Mater. 709 (2015) 530-533.

[10] A. V. Pitukhin, I. G. Skobtsov, The Statistical Catastrophe Theory and Optimal Probability Based Design, Appl. Mech. Mater. 741 (2015) 283-286.

[11] A. V. Pitukhin, V. M. Kostyukevich, I. G. Skobtsov, Energy-absorbing cab support of forest machine, Appl. Mech. Mater. 791 (2015) 290-296. 